\title{
Subsidiariedad en el sector pesquero español: Impacto y efectividad de la política comunitaria de pesca
}

\author{
ENCARNACIÓN CORDÓN LAGARES ${ }^{\text {a }}$ FÉLIX GARCÍA ORDAZ ${ }^{\text {a }}$ \\ a Universidad de Huelva, Facultad de Ciencias Empresariales, Campus de la Merced, Plaza de la \\ Merced, 21071 Huelva, España.E-mail: ecordon@ehu.es, felix@ehu.es
}

\begin{abstract}
RESUMEN
Las políticas basadas en la concesión de subsidios a sectores económicos concretos, y entre ellos al sector pesquero, se han generalizado durante los últimos cincuenta años y a pesar de su dilatada historia, no se ha llegado a alcanzar un consenso sobre su alcance y efectos. El análisis del impacto y efectividad de la política de subvenciones por parte de la UE al sector pesquero español constituye el principal objetivo de este trabajo, para lo cual ha sido necesario contemplar el análisis evolutivo de estos subsidios durante el período 1994-2012. De las diferentes líneas de ayuda al sector pesquero por la UE dos han sido especialmente relevantes: las ayudas a la modernización y las destinadas a la reducción del esfuerzo pesquero mediante el desguace.
\end{abstract}

Palabras clave: Subvenciones, política pesquera.

\section{Subsidiarity in the Spanish Fishing Sector: Impact of the Main EU Policies on Spain}

\begin{abstract}
Policies based on grants to specific economic sectors such as fishery have become widespread in the last fifty years. Despite their long history, there's not a consensus on their scope and effects.

The main objective of this paper is to carry out an analysis of the impact and effectiveness of the EU policies on fisheries subsidies in Spain. To accomplish this task, an analysis of the evolution of these subsidies in the period 1994-2012, was done. The paper highlights the aid for modernization of fishing vessels and reduction in fishing effort by scrapping.
\end{abstract}

Keywords: Subsidies, Fisheries Policy.

Clasificación JEL: F53, H25, Q22, Q28

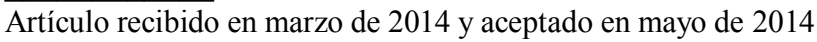

Artículo disponible en versión electrónica en la página www.revista-eea.net, ref. ə-32305 


\section{INTRODUCCIÓN}

La sobreexplotación a la que se ven sometidas muchas de las poblaciones marinas de interés comercial ha sido un tema recurrente en los últimos decenios en la investigación pesquera. El interés por diagnosticar la situación y detectar los factores que la han provocado se ha convertido, por tanto, en elemento predominante. Diferentes políticas de gestión pesquera han sido implementadas con la intención de devolver la sostenibilidad biológica y económica a la actividad, pero en muchos casos los gestores del recurso se han encontrado con un sector altamente dependiente de las ayudas en forma de subsidios y se ha puesto de manifiesto en muchos casos cómo esta estrategia limita o condiciona en muchos casos el éxito de otras políticas de gestión.

En 2012 la Comisión Europea estimó que tres de cada cuatro poblaciones marinas comerciales estaban sobreexplotadas, exhibiendo elevadas concentraciones de sobrepesca en el mediterráneo y el atlántico, con un $82 \%$ y un $63 \%$ de especias amenazadas, respectivamente ${ }^{1}$. Este hecho no hace más que poner de manifiesto el futuro incierto al que se enfrenta la actividad pesquera, amenazada por el importante descenso de las capturas y la pérdida de rentabilidad de las explotaciones pesqueras. Como consecuencia de esto, la UE ha tenido que reorientar de nuevo su política de estructuras pesqueras y dirigir sus esfuerzos hacia la reducción de la flota pesquera comunitaria abriendo para ello líneas de fondos para la concesión de ayudas al desguace sobre todo de los buques más obsoletos $^{2}$.

Ya desde 1983 se han ido adaptando los Programas de Orientación Plurianuales (POP) con objeto de reducir el tamaño de la flota comunitaria para garantizar el ejercicio sostenible de la actividad pesquera. Aunque los POP I (1983-1986) y POP II (1987-1991) no consiguieron reducir el exceso de capacidad de la flota, al menos evitaron el incremento de la capacidad de pesca y pusieron de manifiesto la falta de información fiable sobre el tonelaje y la potencia instalada de la flota comunitaria así como la ausencia de mecanismos de control que asegurasen el fin adecuado de los fondos recibidos por los armadores ${ }^{3}$. El POP III (1992-1996) representó un paso hacia adelante, dividiendo la flota en grupos o segmentos relacionados con las especies objetivo y con las artes de pesca utilizadas. Por último, el POP IV (1997-2001), que seguía la misma orientación que el anterior, fue sustituido por un régimen de entradas y salidas

\footnotetext{
${ }^{1}$ Comunicación de la Comisión-Consulta sobre las posibilidades de pesca para 2012. COM(2011) 298 final.

${ }^{2}$ La Política Estructural de la Pesca nació en 1970 tras la puesta en marcha de una línea de ayudas a la construcción, modernización, comercialización y transformación de las actividades pesqueras con fondos FEOGA.

${ }^{3}$ Comisión Europea (1998).
} 
de buques pesqueros, estando obligados los Estados Miembros (EEMM, en adelante), a partir de enero de 2003, a respetar un régimen muy estricto de entradas y salidas de los buques de su flota para mantener su capacidad pesquera.

Para contribuir a alcanzar un equilibrio compatible con el mantenimientos de los recursos pesqueros y su explotación, así como para reforzar la competitividad del sector y el desarrollo socioeconómico de las áreas geográficas dependientes de la pesca en la UE, en 1994 se creó el Instrumento Financiero de Orientación de la Pesca (IFOP) que gestionó el exceso de capacidad de pesca de la flota comunitaria a través de los Programas de Orientación Plurianuales (POP), cuya intervención más frecuente lo fue financiando medidas para el ajuste del esfuerzo pesquero mediante la puesta en marcha de primas por paralización definitiva del buque, ayudas al desguace y la creación de sociedades mixtas ${ }^{4}$.

En 2007 el IFOP fue sustituido por el Fondo Europeo de Pesca (FEP), más acorde al nuevo principio de sostenibilidad, con unos procedimientos más sencillos y diseñado para reflejar las necesidades de la UE de los 27 , situándose entre sus ejes prioritarios las medidas a favor de la adaptación en el tamaño de la flota pesquera comunitaria mediante la retirada temporal o permanente de buques $^{5}$.

A pesar de los esfuerzos realizados en el último tramo del siglo XX, en la actualidad la sobreexplotación de los recursos pesqueros se ha convertido en un problema de primera magnitud, suponiendo éste la principal amenaza para la continuidad de la actividad pesquera en los países desarrollados ${ }^{6}$. La política basada en subvencionar la reducción del esfuerzo pesquero se ha instrumentalizado en un extenso paquete de medidas, algunas de las cuales no han logrado el objetivo para el que fueron implementadas.

En 2011 el Tribunal de Cuentas Europeo informó sobre la no adecuación de los programas de desguace, los cuales, en opinión del organismo, no se formularon ni se aplicaron correctamente, criticándose también la debida comunicación de los resultados de dichos programas de reducción del esfuerzo pesquero. Por ello, resulta trascendental analizar en qué medida estas políticas de incentivos

\footnotetext{
${ }^{4}$ Reglamento (CEE) n ${ }^{\circ}$ 2080/93 del Consejo, de 20 de julio de 1993 y Reglamento (CE) $\mathrm{n}^{\circ}$ 1263/1999 del Consejo, de 21 de junio de 1999, relativo al Instrumento Financiero de Orientación de la Pesca.

${ }^{5}$ Reglamento (CE) 1198/2006 del Consejo de 27 de julio de 2006 relativo al Fondo Europeo de Pesca.

${ }^{6}$ De hecho, el documento de la FAO (2005) indica que el 17\% de las poblaciones de las que se tienen información están consideradas sobreexplotadas y un $52 \%$ plenamente explotadas. Las principales áreas de pesca con elevados porcentajes (69-77\%) de poblaciones plenamente explotadas son el Atlántico centro-occidental, el Atlántico centro-oriental, el Atlántico noroccidental, el Océano Índico occidental y el Pacífico noroccidental.
} 
basados en subsidios no sólo no han conseguido el objetivo deseado sino que han supuesto un auténtico estímulo para agravarlo.

Como respuesta al resultado de anteriores programas, la nueva política de pesca comunitaria quiere acabar con la dependencia de las subvenciones. Es por ello que, a partir de 2013, el nuevo Fondo Europeo Marítimo y de Pesca (FEMP) eliminará progresivamente las ayudas al desguace, con un periodo de transición hasta 2017.

Este trabajo pretende evidenciar las razones que fundamentan la situación actual, analizando el impacto y efectividad que han tenido las principales políticas pesqueras estructurales de la UE en los últimos veinte años?

El resto del artículo se ha estructurado como sigue: en primer lugar se perfila el contexto pesquero español; a continuación se realiza un estudio de las diferentes medidas basadas en subsidios a la pesca instrumentalizadas durante el período 1994-2012 para el caso español, considerando para ello dos períodos claramente diferenciados: 1994-2006 y 2007-2012. En tercer lugar se analizan las ayudas al desguace de buques pesqueros en el seno de la UE por su relevancia a la hora de argumentar las conclusiones de este estudio, mereciendo un apartado específico. En cuarto lugar se expone la situación en la que se encuentran actualmente estas políticas de incentivos vía subsidios en materia de pesca en la UE y se hará especial hincapié en las perspectivas en un futuro próximo para, finalmente, presentar de manera resumida las principales conclusiones derivadas de este trabajo.

\section{EL CONTEXTO PESQUERO ESPAÑOL}

La flota pesquera española ocupa el tercer lugar respecto a la flota europea, con 10.143 embarcaciones censadas en 2012, el primer lugar respecto al arqueo y el tercer lugar en términos de potencia del motor, lo que demuestra la gran importancia pesquera de España, aportando el mayor número de capturas de entre las flotas de los EEMM ${ }^{8}$. A pesar de ello, y tal y como se muestra en la Tabla 1, el tamaño de la flota pesquera española ha experimentado un notable retroceso en los últimos años. De hecho, se ha producido una caída en el número de buques de aproximadamente el $46 \%$ en el período 1994-2012, que ha

\footnotetext{
${ }^{7}$ En Surís, Varela e Iglesias (2003) se analiza la efectividad de la política estructural en el sector de la pesca en la UE para el período 1994-1999, y en Surís y Varela (2011) para el período 2000-2008. Con anterioridad, Hatcher (2000) ya describió la evolución de la política estructural de la UE para la pesca en el período 1971-1999, pero haciendo especial hincapié en cómo los fondos públicos han logrado tanto el desarrollo como la reestructuración de la flota pesquera comunitaria.

${ }^{8}$ Información suministrada por Eurostat.
} 
provocado una disminución en la capacidad de pesca de aproximadamente el $40 \%$, y en la potencia del $49 \%{ }^{9}$.

El descenso del número de buques es más relevante si cabe en el caso español, ya que el tonelaje medio de las embarcaciones españolas es de 38,2 GT (toneladas de arqueo bruto) mientras que la media de la flota comunitaria está en torno a los 20,2 GT. Algo similar ocurre en cuanto a la potencia de las embarcaciones, pues en el caso español la flota arroja unos valores medios de 86,5 $\mathrm{Kw}$ mientras que para el resto de socios comunitarios es de 77,1 Kw. Sin lugar a dudas, el peso de la potente industria congeladora equipada con buques de gran dimensión ayuda a explicar estas diferencias.

Tabla 1

Evolución de la flota pesquera española 1994-2012

\begin{tabular}{|c|c|c|c|c|c|c|c|}
\hline Años & $\begin{array}{c}\text { Número } \\
\text { buques }\end{array}$ & $\begin{array}{c}\text { Capacidad } \\
(\mathbf{G T})\end{array}$ & $\begin{array}{c}\text { Potencia } \\
\mathbf{( K w )}\end{array}$ & Años & $\begin{array}{c}\text { Número } \\
\text { buques }\end{array}$ & $\begin{array}{c}\text { Capacidad } \\
(\mathbf{G T})\end{array}$ & $\begin{array}{c}\text { Potencia } \\
(\mathbf{K w})\end{array}$ \\
\hline 1994 & 18.917 & 644.226 & 1.714 .490 & 2004 & 14.057 & 491.194 & 1.152 .007 \\
\hline 1995 & 18.385 & 607.493 & 1.631 .154 & 2005 & 13.700 & 487.556 & 1.124 .363 \\
\hline 1996 & 18.151 & 573.385 & 1.539 .862 & 2006 & 13.365 & 482.894 & 1.098 .858 \\
\hline 1997 & 17.978 & 550.336 & 1.470 .596 & 2007 & 13.011 & 470.709 & 1.064 .176 \\
\hline 1998 & 17.978 & 550.340 & 1.470 .597 & 2008 & 11.420 & 461.071 & 1.029 .530 \\
\hline 1999 & 17.321 & 533.331 & 1.381 .730 & 2009 & 11.119 & 439.594 & 979.667 \\
\hline 2000 & 16.678 & 521.838 & 1.332 .708 & 2010 & 10.847 & 414.527 & 934.078 \\
\hline 2001 & 15.436 & 525.847 & 1.301 .073 & 2011 & 10.504 & 398.892 & 900.602 \\
\hline 2002 & 14.924 & 519.539 & 1.260 .178 & 2012 & 10.143 & 387.503 & 877.591 \\
\hline 2003 & 14.398 & 487.137 & 1.177 .218 & - & - & - & - \\
\hline
\end{tabular}

Fuente: Elaboración propia a partir de datos de Eurostat

Se puede afirmar, por tanto, que las capturas totales de la flota española han experimentado un descenso de un 30,2\% en el período 1994-2012. Esta evolución se debe tanto a la disminución de los recursos accesibles como a la fuerte reestructuración de la flota congeladora. En cuanto a la productividad, medida en capturas por barco, la Tabla 2 muestra un máximo en el año $2011(81,88 \mathrm{Tm}$ por buque) y un mínimo en 2004 (54,95 Tm por buque), siendo la media de este indicador para el período analizado de 64,94 $\mathrm{Tm}$ por buque.

Deben ponerse de manifiesto las enormes dificultades motivadas por la gran diversidad de flotas y pesquerías, de dimensiones muy dispares y desarrolladas en entornos muy variables en ecosistemas complejos y donde el impacto socioeconómico de la pesca sobre las poblaciones de pescadores de localidades ribe-

\footnotetext{
${ }^{9}$ Surís y Varela (2011) establecen que no todos los países de la UE han realizado un ajuste de la flota similar. De hecho, algunos EEMM, como España, acometieron ajustes en la capacidad de sus flotas durante el IFOP muy por encima de la media.
} 
reñas es en muchos casos muy determinante a la hora de diseñar mecanismos de gestión de la actividad pesquera, condicionando su efectividad. En este sentido se pronuncia, entre otros, González Laxe (2012), quien destaca que la gran diversidad y heterogeneidad de las modalidades de pesca junto a la elevada atomización y dispersión de los agentes genera varias complejidades, lo cual hace que se requieran muchos datos científicos para adoptar decisiones y se presencien demasiadas luchas de intereses por proponer una acción.

Tabla 2

Productividad de la flota española en el período 1994-2012

\begin{tabular}{|c|c|c|c|c|c|c|c|}
\hline Años & $\begin{array}{c}\text { Capturas/ } \\
\text { Barco }\end{array}$ & $\begin{array}{c}\text { Capturas/ } \\
\text { GT }\end{array}$ & $\begin{array}{c}\text { Capturas/ } \\
\text { Kw }\end{array}$ & Años & $\begin{array}{c}\text { Capturas/ } \\
\text { Barco }\end{array}$ & $\begin{array}{c}\text { Capturas/ } \\
\text { GT }\end{array}$ & $\begin{array}{c}\text { Capturas/ } \\
\text { Kw }\end{array}$ \\
\hline 1994 & 57,39 & 1,69 & 0,63 & 2004 & 54,95 & 1,57 & 0,67 \\
\hline 1995 & 63,58 & 1,92 & 0,72 & 2005 & 56,05 & 1,58 & 0,68 \\
\hline 1996 & 64,08 & 2,03 & 0,76 & 2006 & 55,64 & 1,54 & 0,68 \\
\hline 1997 & 65,90 & 2,15 & 0,81 & 2007 & 56,72 & 1,57 & 0,69 \\
\hline 1998 & 67,31 & 2,20 & 0,82 & 2008 & 80,45 & 1,99 & 0,89 \\
\hline 1999 & 67,20 & 2,18 & 0,84 & 2009 & 68,42 & 1,73 & 0,78 \\
\hline 2000 & 63,96 & 2,04 & 0,80 & 2010 & 68,12 & 1,78 & 0,79 \\
\hline 2001 & 70,99 & 2,08 & 0,84 & 2011 & 81,88 & 2,16 & 0,95 \\
\hline 2002 & 57,07 & 1,64 & 0,68 & 2012 & 74,71 & 1,96 & 0,86 \\
\hline 2003 & 59,52 & 1,76 & 0,73 & - & - & - & - \\
\hline
\end{tabular}

Fuente: Elaboración propia a partir de datos de Eurostat.

Según el Libro Blanco de la Pesca (2009), en el período 1999-2005 las categorías de flota que se han reestructurado más fuertemente han sido las flotas congeladora, artesanal, de arrastre y cerco de las aguas comunitarias y españolas. No obstante, la flota de artes fijos apenas ha revelado una leve reestructuración.

\section{UNA REVISIÓN DEL PAPEL DE LAS SUBVENCIONES AL SECTOR PESQUERO}

Las políticas basadas en otorgar subsidios a sectores económicos como el sector pesquero se han generalizado durante los últimos cincuenta años. No obstante, a pesar de su dilatada historia, no existe un acuerdo único que establezca lo que es una subvención y cómo se puede medir su efecto. Entre los cinco nombres más utilizados para denotar los pagos que realizan los gobiernos al sector pesquero podemos citar los siguientes: subsidios, programas de apoyo, apoyo financiero, asistencia económica y transferencias financieras de los gobiernos.

Recientes estudios de la FAO que analizan el impacto de las subvenciones en el sector pesquero ponen en tela de juicio este sistema soportado en la subsi- 
diariedad, vinculándolo con el fenómeno de la sobrepesca. La UE es también consciente de que determinados mecanismos en forma de ayudas pueden tener un impacto negativo en el medio ambiente, mientras que otras tienen unos efectos positivos directos y contribuyen a reducir la capacidad pesquera. Por ello, es crucial diferenciar las ayudas que contribuyen a la conservación de las especies pesqueras de aquellas que pueden favorecer la sobrecapacidad. A pesar de lo profusamente analizado del asunto, tampoco se ha llegado a alcanzar un consenso sobre su alcance y efectos.

En este sentido, la FAO (1992) atribuyó gran parte la crisis pesquera a la existencia de las subvenciones. Dicho informe puso de manifiesto una seria dificultad a la hora de definir, medir y determinar los efectos de estas subvenciones. Once años más tarde, el informe de la FAO (2003) aportó elementos para conceptualizar a las subvenciones, caracterizándolas como políticas gubernamentales en ayuda de una o más industrias que aportan un beneficio financiero a la industria y que tienen efectos sobre la sostenibilidad, el comercio internacional y el crecimiento económico ${ }^{10}$.

Más precisa es, sin embargo, la definición consagrada en el Acuerdo sobre Subvenciones y Medidas Compensatorias de la Organización Mundial del Comercio (OMC), que considera tres elementos esenciales: i) una contribución financiera o forma de sostenimiento de los ingresos o de los precios, ii) un gobierno o cualquier organismo público en el territorio de un Miembro y iii) la concesión de algún tipo de beneficio.

No existe un único criterio a la hora de clasificar las subvenciones pesqueras; las distintas categorías se solapan sobre todo en función de la naturaleza de la subvención y el propósito de la clasificación. Son especialmente relevantes las realizadas por Milazzo (1998), la Organización para la Cooperación y Desarrollo Económico (OCDE, 2000) y la Cooperación Económica Asía Pacífico $(\text { APEC, 2000) })^{11}$.

Uno de los estudios más citados sobre las subvenciones es el realizado por Milazzo (1998), en el que se aporta una visión crítica acerca de un sector pesquero excesivamente subsidiado. De hecho, indica que mientras la ineficacia en la gestión de pesquerías es la causa que provoca la sobreexplotación, resulta incongruente comprobar la eficacia que se presenta en la gestión respecto a las subvenciones que contribuyen al mantenimiento de la renta del sector.

${ }^{10}$ Sumaila y Pauly (2006) sostienen que los subsidios pesqueros influyen en gran medida en la sostenibilidad de los recursos pesqueros. Así, los subsidios que reducen el coste de las operaciones pesqueras y los que mejoran los ingresos de la pesca, hacen que las empresas sean más rentables.

${ }^{11}$ Cox y Schmidt (2002) realizan una revisión del trabajo de la OCDE sobre subvenciones a la pesca 
Más preciso aún se postula Sumaila (2003) quien sostiene que los subsidios que incrementan los ingresos o reducen los costes de pesca conducen a un aumento marginal en el resultado, lo que aumenta la participación y el esfuerzo de pesca. Sumaila et al. (2010), así como Munro y Sumaila (2002) señalan que las inmensas cantidades destinadas a los subsidios tienen un efecto destructivo sobre la gestión de los recursos pesqueros. También se reconoce por parte de diferentes organizaciones internacionales la importancia del vínculo entre las subvenciones y la gestión pesquera (Porter 2002a y WWF 2002).

El análisis realizado por el Programa de Naciones Unidas para el Medio Ambiente (UNEP) en 2008 revela que la mayoría de las subvenciones tienen el potencial de ser perjudiciales para las poblaciones de peces, sobre todo en ausencia de una gestión eficaz. En realidad, muy pocas pesquerías están sujetas a una gestión lo suficientemente efectiva para asegurar que los subsidios no dañen los recursos pesqueros. De hecho, los subsidios que contribuyen directamente al aumento de la capacidad o el esfuerzo pesquero son algunos de los más dañinos.

El estudio realizado por WWF (2003) al sector pesquero español, uno de los más representativos de la UE, reveló que el 48,4\% del total de las subvenciones económicas otorgadas por el IFOP correspondieron a ayudas con impactos ambientales negativos.

La relación entre subvenciones, sobrecapacidad y sobrepesca se ha reiterado en la Cumbre Mundial sobre el Desarrollo Sostenible (CMDS) celebrada en Johannesburgo (2002), la Conferencia de Ministros de Doha (2001), el documento Código de Conducta para la Pesca Responsable de la FAO (1995), y la Evaluación de Ecosistemas del Milenio (2005) suscitando una especial interés en este tema de investigación. La Consulta técnica de la FAO (2004) sobre la utilización de las subvenciones en el sector pesquero revela que las políticas pesqueras nacionales que utilizan subvenciones son polémicas tanto a nivel nacional como internacional. Por ello, se propone una iniciativa técnica mundial para generar información sobre los efectos observados de las subvenciones en la actividad pesquera.

Una visión general del estado del conocimiento sobre subvenciones a la pesca es ofrecida por Porter (2002b), destacando también WWF (2002), quienes establecen que la poca transparencia de los subsidios pesqueros resulta realmente problemática. Como señalan Prellezo et al. (2012), dado que las cuestiones de la vida real en las pesquerías son tan diversas, para darles respuesta se requiere una diversidad de modelos. De hecho, el modelado de las pesquerías ha sufrido una rápida evolución (Arnason, 2007) ${ }^{12}$.

${ }^{12}$ En este sentido, Cardenete et al.(2013) proponen un análisis que permite conocer el impacto económico de los Fondos Europeos recibidos por la Comunidad Autónoma de Andalucía durante el período 2000-2006, a partir de la matriz de contabilidad social regional del año 2005 $\mathrm{y}$ un modelo de multiplicadores lineales. 


\section{CONSIDERACIONES METODOLÓGICAS PREVIAS}

Para cumplir con el objetivo propuesto, este análisis se ha apoyado en la información suministrada en fishsubsidy.org, proyecto coordinado por la UE con el fin de proporcionar datos relativos a los pagos y beneficiarios de subvenciones a la pesca en todos los EEMM de la UE ${ }^{13}$.

Este trabajo pretende, en primer lugar, identificar las modalidades de subvenciones a la pesca en España para el período 1994-2012, prestando una especial atención al caso de las ayudas destinadas al desguace y ayudas a la modernización de buques pesqueros. Posteriormente se ha llevado a cabo una clasificación de las subvenciones pesqueras utilizando como criterio el potencial impacto en la sostenibilidad de los recursos pesqueros, siendo conscientes de que el efecto de una subvención depende del estado de la pesquería y del sistema de gestión imperante en cada momento.

De hecho, se han utilizado dos clasificaciones: la primera, utilizada entre otros por el proyecto fishsubsidy, divide las subvenciones en buenas, regulares y malas ${ }^{14}$. Esta clasificación se basa en unos indicadores elaborados por el Centro de Investigaciones Pesqueras (CIP) de la Universidad de la Columbia Británica, en el cual "las únicas subvenciones que se consideran buenas son aquellas que favorecen el incremento de las poblaciones de peces con iniciativa de conservación, así como el seguimiento de los volúmenes de capturas gracias a medidas de vigilancia y control que permiten alcanzar una explotación biológica óptima. Entre ellas figuran los programas y servicios de gestión pesquera y las actividades de desarrollo e investigación sobre la pesca".

La segunda clasificación utilizada fue desarrollada por el grupo integrado por representantes de UNEP, la OMC y la OCDE, clasificando las subvenciones como positivas, negativas o neutras, según el impacto ambiental sobre todo en relación con la capacidad de pesca. En este caso, las medidas positivas incluyen el desguace de buques pesqueros, la paralización temporal de las actividades pesqueras y la prima global individual de cese de la actividad. Las medidas negativas incluyen la construcción y modernización de buques y son consideradas medidas neutrales el apoyo específico a los subsectores relacionados con la construcción y modernización de las instalaciones portuarias, transformación y comercialización, así como las medidas más generales tales como proyectos piloto y de demostración y la asistencia técnica.

${ }^{13}$ Los datos ofrecidos por la web www.fishsubsidy.org provienen de la Comisión Europea, que los recoge a su vez de los informes proporcionados por los gobiernos nacionales que administran la PPC.

${ }^{14}$ Esta clasificación utilizada por fishsubsidy se basa en unos indicadores que fueron utilizados en estudios de ámbito genérico (no estrictamente económicos) y en un marco de estudio bastante más amplio. 


\section{ANÁLISIS EVOLUTIVO DE LA POLÍTICA DE SUBVENCIONES A LA PESCA EN ESPAÑA}

El sector pesquero español recibe una considerable ayuda financiera pública a través de los fondos pesqueros de la UE, así como mediante distintas medidas de ayuda y apoyo como, por ejemplo, las exenciones fiscales. Sin embargo, esta situación entra a menudo en contradicción con los objetivos de la PPC (Política Pesquera Común), en especial con la necesidad de reducir el exceso de capacidad y en ocasiones se ha considerado que ha contribuido a agravar los problemas estructurales, en vez de ayudar a resolverlos ${ }^{15}$.

El estudio de González Laxe (2010) incide en el tema afirmando que el porcentaje de incumplimiento de la PPC es muy alto y resulta muy difícil combinar los objetivos de sostenibilidad biológica, económica y social con la situación actual de los recursos, de la demanda y de los precios de los productos pesque$\operatorname{ros}^{16}$.

A pesar de la relevancia del asunto, no es abundante la literatura económica existente en la que se analizan las repercusiones de las subvenciones públicas a la flota en los EEMM. Surís et al. (2002) y Surís y Garza (2005) analizaron 28 y 51 segmentos de flota comunitarios durante los períodos 1994-1999 y 19942001, respectivamente, y concluyeron que aunque las ayudas estructurales al sector pesquero favorecieron el logro de empresas económicamente viables, así como la consecución de los objetivos de cohesión, también ocasionaron efectos negativos en algunos segmentos de flota al incrementar su sobrecapacidad ${ }^{17}$.

A partir de su adhesión a la UE en 1986, España se convirtió en uno de los países que más se beneficiaron de un mayor porcentaje de cofinanciación procedente de los Fondos Estructurales gracias a su consideración como uno de los EEMM con más dificultades para lograr alcanzar niveles medios de desarrollo respecto a sus socios comunitarios. Con una flota pesquera sobredimensionada y amenazada con la pérdida de derechos de pesca en caladeros de terceros países, la integración de España en la UE suponía restablecer el equilibrio de fuerzas en un sector pesquero comunitario que, con anterioridad a 1986, no había experimentado la necesidad urgente de regular el entramado pesquero comunitario a través de una política pesquera comunitaria concreta.

${ }^{15}$ Markus (2010) establece que las políticas pesqueras que ignoran que una gran parte de las subvenciones de combustibles inducen la sobreexplotación, tienden a fomentar la pesca ineficiente e insostenible así como la mala asignación de los fondos públicos.

${ }^{16}$ Hannesson (1998) considera que la gestión pesquera a menudo sufre de una falta de delimitación explícita de los objetivos que se pretenden alcanzar.

${ }^{17}$ Villasante (2010) y Villasante y Sumaila (2010), entre otros, analizan la evolución de la flota pesquera europea desde diferentes perspectivas y sostienen que el problema de la sobrecapacidad es el tema clave que necesita ser abordado en la próxima reforma de la PPC. 
El análisis de las diferentes modalidades de ayuda a la pesca en España y su evolución ofrece una información de gran interés para comprender la situación actual y los efectos que han tenido las principales políticas de la UE. Para ello, en este apartado se analizará, en primer lugar, el período 1994-2006 en el que estuvo vigente el IFOP, que tenía como misión contribuir a alcanzar el equilibrio entre los recursos del sector pesquero y su explotación, con el objetivo de reforzar la competitividad del sector y el desarrollo de las zonas dependientes de la pesca ${ }^{18}$.

Entre los ámbitos de actuación del IFOP se encontraban el ajuste del esfuerzo pesquero, el desarrollo de la acuicultura y la modernización de la flota, entre otros. Posteriormente, se analizará el período 2007-2012 en el que se mantuvo en vigor el FEP que, como su predecesor el IFOP, ha sido creado para el cumplimiento de los objetivos establecidos por la PPC, entre los que se encuentran la adaptación de la capacidad de la flota al estado actual de los recursos así como la mejora de la competitividad del sector y la incorporación de sistemas de gestión de la calidad y el medio ambiente en los procesos de producción, entre otros.

Aunque el FEP ha consagrado muchas de las medidas que ya existían en el marco del IFOP también incluye un conjunto de soluciones innovadoras para responder a las nuevas dificultades del sector pesquero y a las necesidades suscitadas por la reforma de la PPC. De forma resumida, el FEP financia algunos tipos de ayuda que el IFOP no incluía, como las medidas complementarias de los planes de reconstrucción de los stocks, el apoyo a métodos de pesca más selectivos, el incentivo a la diversificación y a la reconversión de zonas de pesca afectadas por las transformaciones experimentadas por el sector, la ayuda para aplicar métodos de acuicultura que tengan un impacto positivo sobre el medio ambiente y la pesca interior.

\subsection{El período entre 1994 y 2006}

La flota pesquera española recibió entre 1994 y 2006 ayudas públicas por un total de 3.728 mill. $€$, de las que un $28 \%$ se destinaron a favorecer la construcción y modernización de buques, mientras que un $11 \%$ fue dedicado al desguace $^{19}$. Además de estas subvenciones, numerosos buques y armadores han disfrutado de exenciones fiscales para la adquisición de gasóleo. Adicionalmente, la UE destinó unos 165 mill. $€$ anuales para garantizar el acceso de su

\footnotetext{
${ }^{18}$ Con la reforma de la PPC (CE, 2002) se dio más importancia a las restricciones del esfuerzo pesquero así como al control de la cuota de una única especie, restringiendo la entrada a las pesquerías mediante restricciones de capacidad y limitaciones de días de mar (Frost y Andersen, 2006).

${ }^{19}$ Según datos de fishsubsidy.org.
} 
flota a las pesquerías de terceros países incentivando la firma de acuerdos de pesca.

La Tabla 3 muestra las modalidades de ayudas a la pesca en España durante el período 1994-1999. Entre las ayudas que experimentaron unos mayores incrementos en dicho período se encuentran las ayudas para prima global individual de cese de actividad, puesta en marcha de proyectos piloto y aumento de la capacidad de transformación. Otro aspecto destacable es que las ayudas destinadas a la protección y desarrollo de los recursos acuáticos, aunque experimentaron un incremento del 160,32\% durante el período 1994-1996, sufrieron un descenso del 68,80\% en el período 1996-1999. Para estas ayudas se ha podido identificar una discrepancia entre las dos clasificaciones utilizadas, ya que mientras que para el CIP de la Universidad de la Columbia Británica estas medidas se catalogan como una ayuda buena, para la UNEP, WWF y OCDE se consideran como neutrales o no claras al considerar que no quedan claramente identificadas las actividades financiadas ${ }^{20}$.

Tabla 3

Modalidades de subvenciones a la pesca en España (período 1994-1999 (mill. €))

\begin{tabular}{|l|c|c|c|c|c|c|c|c|c|}
\hline \multicolumn{1}{|c|}{ Destino de las ayudas } & $\begin{array}{c}\text { Sub- } \\
\text { sector }\end{array}$ & CIP & $\begin{array}{c}\text { UNEP } \\
\text { WWF } \\
\text { OCDE }\end{array}$ & $\mathbf{1 9 9 4}$ & $\mathbf{1 9 9 5}$ & $\mathbf{1 9 9 6}$ & $\mathbf{1 9 9 7}$ & $\mathbf{1 9 9 8}$ & $\mathbf{1 9 9 9}$ \\
\hline Protec.y desarrollo de rec. acuáticos & $\mathrm{M}$ & $\mathrm{B}$ & $\mathrm{N} / \mathrm{NC}$ & $\mathbf{1 , 8}$ & $\mathbf{2 , 6}$ & $\mathbf{4 , 6}$ & $\mathbf{4 , 1}$ & 3,5 & 1,5 \\
\hline Aumento de capac. prod. acuícola & $\mathrm{A}$ & $\mathrm{R}$ & $\mathrm{NG}$ & 1,5 & 0,7 & 0,2 & 4,5 & 8,7 & 7,4 \\
\hline $\begin{array}{l}\text { Certificación de calidad y } \\
\text { etiquetado }\end{array}$ & $\mathrm{C}$ & $\mathrm{R}$ & $\mathrm{N} / \mathrm{NC}$ & $\mathbf{0 , 9}$ & 0,06 & 0,3 & 0,05 & 0,01 & 0,5 \\
\hline Desguace & $\mathrm{E}$ & $\mathrm{R}$ & $\mathrm{P}$ & 14,1 & 100,3 & 35,8 & 26,1 & 42,7 & 14,3 \\
\hline Iniciativas profesionales & $\mathrm{S}$ & $\mathrm{R}$ & $\mathrm{N}$ & - & - & - & 0,1 & - & 0,007 \\
\hline Jubilación anticipada & $\mathrm{S}$ & $\mathrm{R}$ & $\mathrm{N}$ & - & - & 0,01 & 0,03 & 0,05 & - \\
\hline Medidas socioec. sin especificar & $\mathrm{S}$ & $\mathrm{R}$ & $\mathrm{N} / \mathrm{NC}$ & 2,1 & 0,6 & 1,1 & 1,1 & 2,7 & 1,2 \\
\hline Moderniz. de unidades acuícolas & $\mathrm{A}$ & $\mathrm{R}$ & $\mathrm{N} / \mathrm{NC}$ & 5,1 & 4,6 & 5,9 & 5,0 & 12,2 & 13,7 \\
\hline Paralización temporal & $\mathrm{E}$ & $\mathrm{R}$ & $\mathrm{P}$ & 2,0 & 54,6 & - & - & - & 4,6 \\
\hline Prima global indiv. cese de actividad & $\mathrm{E}$ & $\mathrm{R}$ & $\mathrm{P}$ & - & - & 0,01 & 0,4 & 0,6 & 1,1 \\
\hline Proyectos piloto & $\mathrm{E}$ & $\mathrm{R}$ & $\mathrm{N} / \mathrm{NC}$ & - & - & 0,05 & 1,1 & 0,8 & 1,3 \\
\hline Actividades de promoción & $\mathrm{C}$ & $\mathrm{M}$ & $\mathrm{N} / \mathrm{NC}$ & 0,6 & 3,2 & 3,6 & 7,1 & 8,1 & 7,8 \\
\hline Aumento capac.de transformación & $\mathrm{T}$ & $\mathrm{M}$ & $\mathrm{N} / \mathrm{NC}$ & 3,7 & 3,3 & 13,1 & 29,7 & 33,7 & 50,8 \\
\hline Construc. nuevos buques & $\mathrm{E}$ & $\mathrm{M}$ & $\mathrm{NG}$ & 21,9 & 30,8 & 72,8 & 75,6 & 92,7 & 124,5 \\
\hline Construc. nuevos equip. portuarios & $\mathrm{P}$ & $\mathrm{M}$ & $\mathrm{N}$ & 10,4 & 6,0 & 3,0 & 14,3 & 14,2 & 14,3 \\
\hline $\begin{array}{l}\text { Exportac./Asignación a otras } \\
\text { áreas/Traspaso país tercero }\end{array}$ & $\mathrm{E}$ & $\mathrm{M}$ & $\mathrm{N}$ & 7,4 & 3,9 & - & - & - & - \\
\hline
\end{tabular}

${ }^{20}$ Para las ayudas de prima global individual de cese de actividad y de puesta en marcha de proyectos piloto los porcentajes indicados recogen el incremento experimentado por las ayudas durante el período 1996-1999. 
Tabla 3 (continuación)

Modalidades de subvenciones a la pesca en España

(período 1994-1999 (mill. €))

\begin{tabular}{|l|c|c|c|c|c|c|c|c|c|}
\hline \multicolumn{1}{|c|}{ Destino de las ayudas } & $\begin{array}{c}\text { Sub- } \\
\text { sector }\end{array}$ & CIP & $\begin{array}{c}\text { UNEP } \\
\text { WWF } \\
\text { OCDE }\end{array}$ & $\mathbf{1 9 9 4}$ & $\mathbf{1 9 9 5}$ & $\mathbf{1 9 9 6}$ & $\mathbf{1 9 9 7}$ & $\mathbf{1 9 9 8}$ & $\mathbf{1 9 9 9}$ \\
\hline Modernización de buques existentes & $\mathrm{E}$ & $\mathrm{M}$ & $\mathrm{NG}$ & $\mathbf{4 , 7}$ & $\mathbf{9 , 0}$ & 16,0 & 12,4 & 19,8 & 23,7 \\
\hline Moderniz. equipamientos portuarios & $\mathrm{P}$ & $\mathrm{M}$ & $\mathrm{N}$ & 1,5 & 0,8 & 0,7 & 1,8 & 1,8 & 1,5 \\
\hline Moderniz. establec. comercialización & $\mathrm{C}$ & $\mathrm{M}$ & $\mathrm{N} / \mathrm{NC}$ & $\mathbf{4 , 9}$ & 3,4 & 8,4 & 5,5 & 9,1 & 8,9 \\
\hline $\begin{array}{l}\text { Moderniz. unidades de } \\
\text { transformación }\end{array}$ & $\mathrm{T}$ & $\mathrm{M}$ & $\mathrm{N} / \mathrm{NC}$ & 9,2 & 21,8 & 19,3 & 3,8 & 9,9 & 16,3 \\
\hline Otras medidas de transf. y comerc. & $\mathrm{T} / \mathrm{C}$ & $\mathrm{M}$ & $\mathrm{N} / \mathrm{NC}$ & 4,5 & 13,2 & 4,1 & 14,1 & 9,8 & 9,0 \\
\hline Sociedades mixtas & $\mathrm{E}$ & $\mathrm{M}$ & $\mathrm{NC}$ & $\mathbf{4 7 , 7}$ & 35,2 & 6,3 & 33,3 & 8,8 & 3,3 \\
\hline Sociedades mixtas temporales & $\mathrm{E}$ & $\mathrm{M}$ & $\mathrm{N}$ & 5,9 & 5,3 & 9,1 & 9,4 & 5,6 & 6,6 \\
\hline Total general & & & & $\mathbf{1 5 0}$ & $\mathbf{3 0 0}$ & $\mathbf{2 0 5}$ & $\mathbf{2 5 0}$ & $\mathbf{2 8 5}$ & $\mathbf{3 4 3}$ \\
\hline
\end{tabular}

Nota. Subsector: Acuicultura (A), Comercialización (C), Extractivo (E), Investigación (I), Social (S), Portuario (P), Medioambiental (M), Transformación (T), Transformación/Comercialización (T/C).

Clasificación CIP Columbia: Buena (B), Mala (M), Regular (R).

Clasificación UNEP, WWF y OCDE: Positivo (P), Negativo (NG), Neutral (N), No claro (NC).

Fuente: Elaboración propia a partir de los datos de fishsubsidy.org.

En definitiva, siguiendo la clasificación del CIP, mientras que las ayudas consideradas como buenas se redujeron un $18,79 \%$, las ayudas consideradas como regulares y malas se incrementaron un $70,27 \%$ y $142,13 \%$ en el período 1994-1999, respectivamente. Siguiendo la clasificación de UNEP, WWF y OCDE las ayudas catalogadas como positivas se incrementaron un $22,98 \%$ y las consideradas como neutrales o no claras y las negativas se incrementaron un $57,55 \%$ y un $454,54 \%$, respectivamente.

A pesar de la divergencia existente, en algunas ayudas queda patente la similitud en su clasificación, como es el caso de las ayudas a la construcción de nuevos buques, modernización de los buques existentes y las ayudas a pescadores jóvenes que son consideradas como malas o negativas.

La Tabla 4 muestra la evolución de las acciones estructurales en el sector pesquero durante el período 2000-2006 considerando las diferentes modalidades de ayuda a la pesca en España. Las ayudas que experimentaron unos mayores incrementos en dicho período fueron las ayudas al desguace, jubilación anticipada y modernización de unidades acuícolas existentes.

En este subperíodo, mientras que para el CIP las modalidades de ayudas clasificadas como malas y regulares se redujeron un $53,11 \%$ y un $29,58 \%$, respectivamente, las consideradas buenas lo hicieron un $46,21 \%$ debido principalmente a la reducción que en el año 2006 experimentó la modalidad de ayuda de protección y desarrollo de los recursos acuáticos que pasó de 3 mill. $€$ en el año 2000 a tan sólo 0,2 mill. $€$ en 2006. UNEP, WWF y OCDE consideran que las 
ayudas positivas se redujeron un $48,95 \%$, mientras que las negativas y las neutrales o no claras lo hicieron un $68,08 \%$ y un $24,36 \%$, respectivamente.

\section{Tabla 4}

Modalidades de ayudas a la pesca en España (período 2000-2006, (mill. €))

\begin{tabular}{|c|c|c|c|c|c|c|c|c|c|c|}
\hline Destino de las ayudas & $\begin{array}{l}\text { Sub- } \\
\text { sect }\end{array}$ & CIP & $\begin{array}{l}\text { UNEP, } \\
\text { WWF, } \\
\text { OCDE }\end{array}$ & 2000 & 2001 & 2002 & 2003 & 2004 & 2005 & 2006 \\
\hline Asistencia técnica & 1 & $B$ & $\mathrm{~N} / \mathrm{NC}$ & 4,3 & 5,8 & 5,5 & 6,9 & 5,8 & 6,4 & 3,7 \\
\hline $\begin{array}{l}\text { Protec. y desarr. de recursos } \\
\text { acuáticos }\end{array}$ & M & B & $\mathrm{N} / \mathrm{NC}$ & 3,1 & 6,7 & 3,3 & 5,6 & 2,2 & 7,3 & 0,2 \\
\hline $\begin{array}{l}\text { Aumento capac.de produc. } \\
\text { acuícola }\end{array}$ & A & $\mathrm{R}$ & NG & 4,7 & 13,3 & 16,6 & 1,6 & 11,3 & 17,3 & 7,5 \\
\hline Desguace & $E$ & $\mathrm{R}$ & $\mathrm{P}$ & 8,3 & 25,3 & 11,6 & 24,4 & 28,9 & 32,6 & 33,9 \\
\hline Iniciativas profesional & $S$ & $\mathrm{R}$ & $\mathrm{N}$ & 6,1 & 13,0 & 10,4 & 13,5 & 8,7 & 8,6 & 10,3 \\
\hline Jubilación anticipada & $S$ & $\mathrm{R}$ & $\mathrm{N}$ & 0,03 & 0,4 & 0,02 & 0,6 & 0,9 & 1,9 & 0,7 \\
\hline $\begin{array}{l}\text { Modern. Unid. acuícolas } \\
\text { existentes }\end{array}$ & $A$ & $\mathrm{R}$ & $\mathrm{N} / \mathrm{NC}$ & 0,2 & 15,1 & 11,2 & 20,9 & 13,5 & 17,9 & 8,0 \\
\hline $\begin{array}{l}\text { Paraliz. temporal y otras } \\
\text { indemn. }\end{array}$ & $E$ & $\mathrm{R}$ & $\mathrm{P}$ & 85,1 & 77,1 & 34,4 & 61,8 & 6,2 & 24,4 & 12,4 \\
\hline $\begin{array}{l}\text { Prima reorient. laboral } \\
\text { (diversific.) }\end{array}$ & $S$ & $\mathrm{R}$ & $\mathrm{N}$ & - & - & - & 0,09 & 0,3 & 587,0 & 0,2 \\
\hline $\begin{array}{l}\text { Prima global individual de } \\
\text { cese }\end{array}$ & $E$ & $\mathrm{R}$ & $P$ & - & 0,3 & 0,9 & 0,8 & 0,3 & 1,2 & 1,3 \\
\hline $\begin{array}{l}\text { Proyectos piloto o de } \\
\text { demostración }\end{array}$ & $E$ & $\mathrm{R}$ & $\mathrm{N} / \mathrm{NC}$ & 4,1 & 7,7 & 7,7 & 4,9 & 29,0 & 34,7 & 1,7 \\
\hline Actividades de promoción & $\mathrm{C}$ & $\mathrm{M}$ & $\mathrm{N} / \mathrm{NC}$ & 6,7 & 16,9 & 5,4 & 16,6 & 7,9 & 9,6 & 16,6 \\
\hline $\begin{array}{l}\text { Aumento de capac. } \\
\text { transformación }\end{array}$ & $\mathrm{T}$ & M & $\mathrm{N} / \mathrm{NC}$ & 36,8 & 54,3 & 46,5 & 61,9 & 67,5 & 41,8 & 16,2 \\
\hline $\begin{array}{l}\text { Ayuda de pescadores } \\
\text { jóvenes }\end{array}$ & $\mathrm{S}$ & M & NG & - & - & - & 34,7 & 0,003 & 36,0 & - \\
\hline Construc. de nuevos buques & $E$ & M & NG & 52,2 & 83,1 & 217,6 & 41,4 & 48,8 & 1,6 & - \\
\hline $\begin{array}{l}\text { Construc. nuevos equip. } \\
\text { portuarios }\end{array}$ & $\mathrm{P}$ & M & $\mathrm{N}$ & 19,2 & 20,4 & 7,9 & 17,7 & 9,4 & 15,0 & 10,0 \\
\hline $\begin{array}{l}\text { Construc. nuevos establec. } \\
\text { comercial. }\end{array}$ & $\mathrm{C}$ & M & $\mathrm{N} / \mathrm{NC}$ & 5,1 & 16,3 & 12,2 & 12,7 & 9,6 & 9,7 & 1,4 \\
\hline $\begin{array}{l}\text { Exportación/ } \\
\text { Traspaso país tercer }\end{array}$ & $E$ & M & $\mathrm{N}$ & - & - & - & - & - & - & 0,1 \\
\hline $\begin{array}{l}\text { Modernización de buques } \\
\text { existentes }\end{array}$ & $E$ & M & NG & 5,4 & 14,1 & 13,3 & 13,0 & 14,7 & 7,0 & 12,2 \\
\hline $\begin{array}{l}\text { Moderniz. equip. portuarios } \\
\text { existente }\end{array}$ & $\mathrm{P}$ & M & $\mathrm{N}$ & 4,1 & 10,0 & 7,8 & 9,5 & 8,9 & 4,3 & 4,2 \\
\hline $\begin{array}{l}\text { Moderniz. establec. de } \\
\text { comercializ. }\end{array}$ & $\mathrm{C}$ & M & $\mathrm{N} / \mathrm{NC}$ & 4,1 & 8,9 & 8,8 & 10,1 & 14,8 & 6,4 & 2,9 \\
\hline $\begin{array}{l}\text { Moderniz. Unid. transform. } \\
\text { existente }\end{array}$ & $\mathrm{T}$ & $M$ & $\mathrm{~N} / \mathrm{NC}$ & 12,9 & 14,1 & 7,9 & 12,7 & 13,2 & 11,5 & 3,8 \\
\hline Pesca costera artesanal & $E$ & $M$ & $\mathrm{~N} / \mathrm{NC}$ & - & 0,1 & 0,4 & 1,7 & 4,3 & 3,3 & 2,1 \\
\hline Sociedades mixtas & $E$ & M & NC & 2,6 & 3,8 & 10,7 & 7,7 & 11,3 & 0,7 & - \\
\hline Total general & - & - & - & 265 & 407 & 441 & 347 & 318 & 265 & 150 \\
\hline
\end{tabular}

Nota. Subsector: Acuicultura (A), Comercialización (C), Extractivo (E), Investigación (I), Social (S), Portuario (P), Medioambiental (M), Transformación (T), Transformación/Comercialización (T/C).

Clasificación CIP Columbia: Buena (B), Mala (M), Regular (R).

Clasificación UNEP, WWF y OCDE: Positivo (P), Negativo (NG), Neutral (N), No claro (NC).

Fuente: Elaboración propia a partir de los datos de fishsubsidy.org. 
La flota pesquera española recibió entre 1994 y 2006 ayudas públicas para desguace por un total 398 mill. $€$ procedentes del IFOP, de las cuales 271 mill. $€$ eran fondos procedentes del presupuesto comunitario y 127 mill. $€$ procedían de la cofinanciación aportada por los EEMM.

En definitiva, el período 1994-2006 conoció dos fases tremendamente diferenciadas: un primer subperíodo 1994-1999 de expansión del esfuerzo financiero destinado a los subsidios a la pesca, con un incremento del $127,85 \%$, y una segunda fase, durante el subperíodo 2000-2006 en la que se invierte esta tendencia drásticamente, con una reducción del 43,29\%. Este nuevo enfoque en la política de subvenciones al sector pesquero supuso un tremendo cambio en la concepción tradicional que hasta entonces había tenido la pesca por parte de los EEMM.

\subsection{El período entre 2007 y 2012}

Los instrumentos con los que cuentan los Fondos Estructurales para el nuevo período de programación 2007-2013 sufrieron muchos cambios respecto a los contemplados en el período anterior a raíz de la nueva orientación y redefinición de la política de cohesión europea y del nuevo contexto geopolítico tras la ampliación de la UE a los países del este ${ }^{21}$.

Para el período 2007-2013 se sustituyó el IFOP por el FEP que ya no se articula dentro de los Fondos estructurales de la UE sino que se encuadra en la nueva categoría "Conservación y Gestión de los Recursos Naturales", por lo que la relación entre el desarrollo económico del sector pesquero y el respeto por los recursos y el medioambiente marino adquieren mayor relevancia. El FEP, como sucedía con el IFOP, se crea para lograr los objetivos consagrados en la PPC de adaptación de la capacidad de la flota al estado actual de los recursos y de mejora de la competitividad del sector incorporando sistemas de gestión de calidad y medio ambiente en los procesos de producción, la innovación y el desarrollo de nuevas técnicas respetuosas con el medio ambiente y la seguridad del trabajo a bordo.

En lo relativo a las ayudas a la reestructuración de la flota, el FEP conserva el espíritu de cambio ya introducido en el Reglamento del IFOP a partir del 1 de enero de 2005. Las ayudas a la renovación de la flota, que ya para entonces habían dejado de existir tampoco están presentes en el marco del FEP. Sería paradójico financiar la construcción de nuevos buques más potentes sabiendo que la flota pesquera tiene una capacidad excesiva en relación con los recursos disponibles ${ }^{22}$.

${ }^{21}$ CE (2009a).

${ }^{22}$ Un buque en actividad sólo puede beneficiarse de ayudas europeas en el caso de necesitar transformaciones o equipamientos destinados a mejorar la seguridad a bordo, la calidad de las 
Con el Reglamento (CE) 1198/2006 de 27 de julio de 2006 se establecen cinco ejes de acción, clasificando los distintos tipos de medidas que podrán financiarse con cargo al FEP:

Eje 1: Medidas de adaptación de la flota pesquera comunitaria.

Eje 2: Acuicultura, pesca interior, transformación y comercialización de productos de la pesca y de la acuicultura.

Eje 3: Medidas de interés público.

Eje 4: Desarrollo sostenible de las zonas de pesca.

Eje 5: Asistencia técnica.

Por tanto, el principal objetivo del programa operativo es proporcionar ayuda financiera para facilitar la aplicación de la PPC y apoyar las reestructuraciones necesarias mediante la aplicación de medidas orientadas a garantizar el ejercicio sostenible de la actividad pesquera y a diversificar las actividades económicas en las zonas dependientes de la pesca. Las medidas de este fondo se adaptan a las nuevas necesidades que presentan el sector pesquero y acuícola y las comunidades costeras dedicadas a la pesca. De hecho, se trata de contribuir a reducir la presión que ejerce la pesca para que puedan recuperarse las poblaciones explotadas y fomentar la utilización de equipos y prácticas más ecológicos en los sectores de la pesca y la acuicultura así como en la preparación y comercialización de los productos pesqueros. Evidentemente, se pretende ayudar a las regiones más afectadas por la pérdida de empleo, concediéndoles ayudas para favorecer su diversificación ${ }^{23}$.

En 2009, el segundo Libro Verde sobre la reforma de la PPC (Comisión Europea, 2009b) reconoce el fracaso relativo de la PPC, debido a la sobrepesca que caracteriza a la mayor parte de las pesquerías de la UE, lo cual se ha vinculado principalmente a la excesiva capacidad de la flota pesquera. De hecho, González Laxe (2002 y 2010), Khalilian et al. (2010) y Penas (2002 y 2008) también coinciden con el diagnóstico aportado por la Comisión ${ }^{24}$.

El FEP cuenta, para el periodo 2007-2013, con un presupuesto global para todos los EEMM de 4.305 mill. $€$, siendo España el país de la UE con un mayor

capturas, la selectividad de la pesca o para sustituir un motor que consuma demasiado combustible, con condiciones muy estrictas y siempre que no aumente la potencia y la capacidad pesquera del buque.

${ }^{23}$ Cordón y García (2010) sostienen que la reconversión o diversificación socioeconómica en el sector pesquero resulta compleja por diferentes motivos, entre los que se encuentran las dificultades propias de las zonas dependientes de la pesca y a la existencia de una escasa movilidad profesional de los pescadores.

${ }^{24}$ Surís et al. (2011) pronostican que la futura PPC no sufrirá cambios sustanciales, ni en sus principios fundamentales ni con respecto a sus objetivos y ejes prioritarios. 
porcentaje asignado del FEP, con un 26,29\% (1.132 mill. €) de financiación global $^{25}$.

La Tabla 5 resume el desglose del FEP en España por ejes prioritarios. Los ejes con unos mayores porcentajes asignados del FEP son el Eje 1 (medidas de adaptación de la flota pesquera comunitaria), Eje2 (acuicultura) y Eje 3 (medidas de interés público) con unos porcentajes del 35,61\%, 30,41\% y 27,96\%, respectivamente.

\section{Tabla 5}

Desglose del FEP en España por ejes prioritarios

\begin{tabular}{|c|c|c|c|c|c|}
\hline & Eje 1 & Eje 2 & Eje 3 & Eje 4 & Eje 5 \\
\hline Importe & 403.067 .965 & 344.241 .335 & 316.510 .945 & 49.336 .048 & 18.734 .619 \\
\hline$\%$ & $35,61 \%$ & $30,41 \%$ & $27,96 \%$ & $4,36 \%$ & $1,66 \%$ \\
\hline
\end{tabular}

Fuente: Informe anual sobre la aplicación del FEP (2007).

El análisis comparativo del importe de la ayuda comunitaria para la reestructuración del sector pesquero español durante el período 1994-2013 muestra la enorme reducción del peso de dichas ayudas (véase la Tabla 6). Respecto de la última convocatoria 2000-2006, para el período 2007-2013 el importe de las ayudas se ha visto reducido un $48,4 \%$. A pesar del enorme esfuerzo financiero de la UE durante el período 1994-2006, poniendo en marcha programas de incentivos para la reducción del tamaño de la flota comunitaria, los resultados no han sido todo lo satisfactorios que cabría esperar. La tendencia para este tipo de ayudas se verá caracterizada por un cambio en el rumbo de la política de estructuras pesqueras en la UE. El debate sobre la eficacia de las medidas implementadas está servido.

En apartados siguientes se intentará arrojar luz sobre algunas de estas cuestiones ejemplificándolo con el caso concreto de las ayudas al desguace de la flota implementadas en los últimos años.

\section{Tabla 6}

Importe de las ayudas comunitarias a la pesca en España entre los períodos 1994-1999, 2000-2006 y 2007-2013 (Euros)

\begin{tabular}{|l|c|c|c|c|c|c|}
\hline & $\mathbf{1 9 9 4 - 1 9 9 9}$ & $\mathbf{2 0 0 0 - 2 0 0 6}$ & $\mathbf{2 0 0 7 - 2 0 1 3}$ & $\begin{array}{c}\text { Variación 94-99/ } \\
\mathbf{0 0 - 0 6}\end{array}$ & $\begin{array}{c}\text { Variación 94-99/ } \\
\mathbf{0 7 - 1 3}\end{array}$ & $\begin{array}{c}\text { Variación 00-06/ } \\
\mathbf{0 7 - 1 3}\end{array}$ \\
\hline España & 1.534 .582 .257 & 2.193 .728 .514 & 1.131 .890 .912 & $42,95 \%$ & $-26,24 \%$ & $-48,40 \%$ \\
\hline
\end{tabular}

Fuente: Elaboración propia a partir de los datos de Subsidy.org.

${ }^{25}$ Por acuerdo de la Conferencia Sectorial de 10 de abril de 2007, se establecieron los criterios de reparto de los fondos y la distribución entre Comunidades Autónomas para el período 20072013. 


\section{EL CASO DE LAS AYUDAS AL DESGUACE}

La UE ha adolecido tradicionalmente de una reglamentación suficientemente clara sobre el tratamiento de los derechos de pesca (cancelación, transferencia o venta) cuando los buques pesqueros son desguazados con ayudas públicas. Este es un problema de primera magnitud en cuanto a la gestión óptima de la actividad pesquera, pues los reglamentos comunitarios no especifican de manera nítida y objetiva el papel que deben desempeñar los derechos de pesca en la adaptación de la capacidad pesquera a las posibilidades de pesca.

Así, mientras el reglamento de la PPC contempla la retirada de la licencia de pesca y de las autorizaciones de pesca cuando los buques son desguazados con ayudas públicas, restando de los límites totales de capacidad pesquera la capacidad subyacente en términos de arqueo bruto y potencia, para el caso de la asignación de las cuotas de pesca a los EEMM no se tienen en cuenta las licencias de pesca y la capacidad pesquera de los buques que han sido retirados ${ }^{26}$.

De hecho, estas cuotas no pueden retirarse permanentemente, ya que los EEMM pueden asignarlas a otros buques pesqueros y utilizar, por ejemplo, sistemas de cuotas transferibles ${ }^{27}$. Por tanto no ha existido nunca una definición clara de qué derechos de pesca (autorizaciones y licencias) deben retirarse cuando los buques se desguazan con ayudas públicas ${ }^{28}$.

Esta obligación de retirar derechos de pesca, que figura en el reglamento de la PPC, se contradice con los reglamentos anuales de los Totales Anuales de Captura (TAC), que permiten la reasignación de los derechos de pesca de dichos buques en determinados casos ${ }^{29}$.

Así, mientras algunos EEMM como Dinamarca y España permiten que determinados derechos de pesca se transfieran a otros buques pesqueros o a otros pescadores, en otros como en Francia y Polonia los cancelan. Los resultados del análisis permiten también evidenciar la deficiente reglamentación de las ayudas al desguace, ya que se ha permitido a los beneficiarios de ayudas públicas para el desguace de buques transferir los correspondientes derechos o cuotas de pesca a través de una transferencia de recursos adicionales que proporciona a dichos beneficiarios. De hecho, el reglamento del FEP no recoge la posibilidad de reconocer estos recursos adicionales a la hora de establecer los porcen-

\footnotetext{
${ }^{26}$ Artículo 11, apartado 3, del Reglamento (CE) no 2371/2002 del Consejo.

${ }^{27}$ Arnason (2002) ofrece una valoración del proceso de puesta en marcha de sistemas ITQ (Cuotas Individuales Transferibles).

${ }^{28}$ Tribunal de Cuentas Europeo (2011).

${ }^{29}$ Reglamento (CE) $n^{\circ} 40 / 2008$ del Consejo, el Reglamento (CE) no 43/2009 del y el Reglamento (UE) $n^{\circ}$ 53/2010 del Consejo.
} 
tajes de las ayudas públicas para el desguace de buques pesqueros ${ }^{30}$.

El análisis de la programación 2007-2010 se resume en la Tabla 7 donde se muestra que durante dicho período España ha recibido más de 148 mill. $€$ procedentes de ayudas al desguace de buques pesqueros, siendo la variación experimentada en dicho período del $482,83 \%$.

Tabla 7

Ayudas al desguace durante el período 2007-2010 (mill. €)

\begin{tabular}{|c|c|c|c|c|c|c|}
\hline Año & $\mathbf{2 0 0 7}$ & $\mathbf{2 0 0 8}$ & $\mathbf{2 0 0 9}$ & $\mathbf{2 0 1 0}$ & Total & $\begin{array}{c}\text { Variación } \\
\mathbf{2 0 0 7 / 2 0 1 0}\end{array}$ \\
\hline Ayudas al desguace & 11,1 & 10,0 & 61,8 & 65,1 & 148,1 & $482,83 \%$ \\
\hline
\end{tabular}

Fuente: Elaboración propia.

La Tabla 8 muestra la contribución de los fondos IFOP al desguace según los segmentos de flota, destacando que los segmentos de flota que más se han beneficiado de ayudas del IFOP para el desguace respecto al total de buques desguazados en dichos segmentos han sido aquellos que utilizan redes de cerco $(33 \%)$, seguidos por los que utilizan como modalidad principal redes de arrastre $(32 \%)$.

Tabla 8

Contribución de los fondos del IFOP al desguace de buques por segmento de flota

\begin{tabular}{|c|c|c|c|c|c|c|c|c|c|}
\hline & \multicolumn{3}{|c|}{$\begin{array}{c}\text { Buques desguazados } \\
\text { con ayudas del IFOP }\end{array}$} & \multicolumn{3}{c|}{ Desguace total } & \multicolumn{3}{c|}{$\begin{array}{c}\% \text { of buques desguazados } \\
\text { IFOP/total desguazados }\end{array}$} \\
\hline $\begin{array}{c}\text { Categoría } \\
\text { principal }\end{array}$ & $\begin{array}{c}\text { Toneladas } \\
(\mathbf{G T})\end{array}$ & $\begin{array}{c}\text { Potencia } \\
(\mathbf{K w})\end{array}$ & $\begin{array}{c}\text { Buques } \\
\left(\mathbf{N}^{\circ}\right)\end{array}$ & $\begin{array}{c}\text { Toneladas } \\
(\mathbf{G T})\end{array}$ & $\begin{array}{c}\text { Potencia } \\
(\mathbf{K w})\end{array}$ & $\begin{array}{c}\text { Buques } \\
\left(\mathbf{N}^{\circ}\right)\end{array}$ & $\begin{array}{c}\text { Toneladas } \\
(\mathbf{G T})\end{array}$ & $\begin{array}{c}\text { Potencia } \\
(\mathrm{Kw})\end{array}$ & $\begin{array}{c}\text { Buques } \\
\left(\mathbf{N}^{\circ}\right)\end{array}$ \\
\hline $\begin{array}{c}\text { Artes de } \\
\text { trampa }\end{array}$ & 28 & 217 & 7 & 764 & 3.756 & 132 & $4 \%$ & $6 \%$ & $5 \%$ \\
\hline $\begin{array}{c}\text { Líneas } \\
\text { anzuelos }\end{array}$ & 13.359 & 37.925 & 230 & 40.837 & 112.113 & 1.160 & $33 \%$ & $34 \%$ & $20 \%$ \\
\hline Rastras & 5 & 44 & 2 & 402 & 3.635 & 170 & $1 \%$ & $1 \%$ & $1 \%$ \\
\hline $\begin{array}{c}\text { Redes de } \\
\text { arrastre }\end{array}$ & 90.686 & 216.805 & 554 & 202.775 & 525.149 & 1.710 & $45 \%$ & $41 \%$ & $32 \%$ \\
\hline $\begin{array}{c}\text { Redes de } \\
\text { cerco }\end{array}$ & 15.180 & 62.515 & 298 & 49.498 & 182.556 & 902 & $31 \%$ & $34 \%$ & $33 \%$ \\
\hline $\begin{array}{c}\text { Redes de } \\
\text { enmalle y } \\
\text { enredo }\end{array}$ & 12.313 & 44.906 & 651 & 26.072 & 134.299 & 3.670 & $47 \%$ & $33 \%$ & $18 \%$ \\
\hline Desconocida & 0 & 0 & 0 & 17 & 53 & 2 & $0 \%$ & $0 \%$ & $0 \%$ \\
\hline Total & $\mathbf{1 3 1 . 5 7 1}$ & $\mathbf{3 6 2 . 4 1 2}$ & $\mathbf{1 . 7 4 2}$ & $\mathbf{3 2 0 . 3 6 5}$ & $\mathbf{9 6 1 . 5 6 1}$ & $\mathbf{7 . 7 4 6}$ & $\mathbf{4 1 \%}$ & $\mathbf{3 8 \%}$ & $\mathbf{2 2 \%}$ \\
\hline
\end{tabular}

Fuente: Elaboración propia a partir de los datos del Registro de flota de la UE.

${ }^{30}$ En el caso de España, una de las condiciones para acceder a las ayudas al desguace de buques pesqueros era que el buque hubiese faenado durante al menos 90 días en cada uno de los últimos dos años antes de la fecha de la solicitud o bien hubiese faenado durante al menos 120 días en el año anterior a la fecha de la solicitud. 
Entre las Comunidades Autónomas que han recibido una mayor dotación financiera en ayudas al desguace, en el período 1994-2006, se encuentran Galicia con el $31,98 \%$ de dicho montante (127 mill. $€$ ), seguida de Andalucía que recibió el 16,27\% (65mill. €), es decir, dos de las regiones con mayores índices de sobrecapacidad de flota ${ }^{31}$.

Un elemento de discusión relevante lo constituye el uso inadecuado o perverso, en ciertas ocasiones, de algunos instrumentos de la política estructural como es el caso de las ayudas a desguaces de buques pesqueros. En este sentido, se ha podido detectar la existencia de buques que percibieron ayudas públicas con objeto de desguazarlos y reducir la capacidad de la flota al poco tiempo después de haber recibido subvenciones para su modernización. En la Figura 1 se muestra el número de buques que recibieron ayudas a la modernización y posterior desguace durante el período 1998-2011, con un total de 326 buques españoles. El montante total recibido fue de 7,7 mill. $€$ en ayudas a la modernización y 95,3 mill. € para el desguace, de los que 6,2 mill. $€$ y 67,5 mill. $€$ eran ayudas de la UE a la modernización y desguace, respectivamente. Si se tiene en cuenta el tiempo transcurrido desde que se concede la ayuda a la modernización y posteriormente al desguace, algo más de la mitad de los buques (51\%) recibieron ambos tipos de ayudas en un intervalo igual o inferior a los 5 años, pudiendo este análisis identificar a 10 buques a los que se les concedió ambos tipos de ayuda en un lapso de tiempo igual o inferior al año.

Figura 1

Número de buques con ayuda a la modernización y al desguace en el período 1998-2011

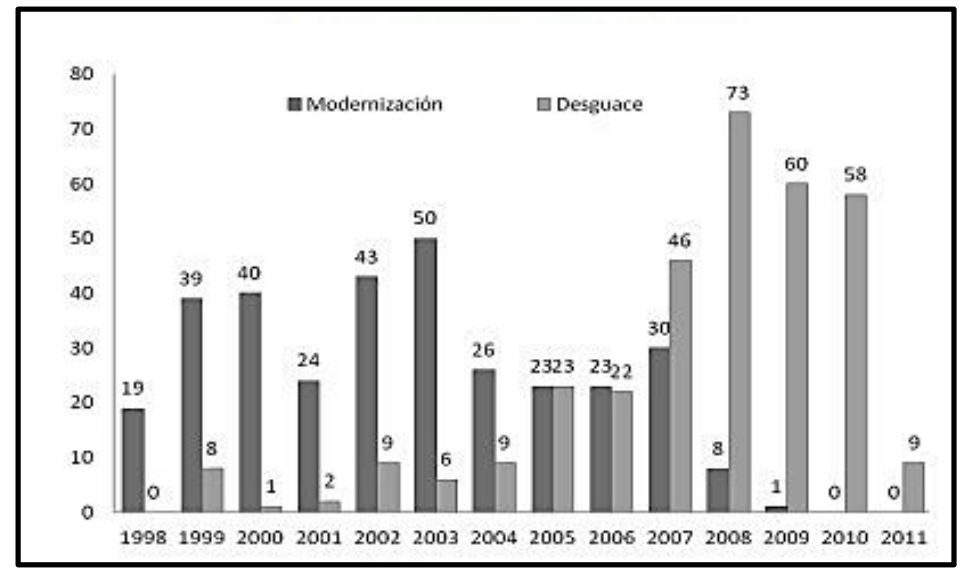

Fuente: Elaboración propia a partir de los datos de los datos de Subsidy.org.

${ }^{31}$ Fishsubsidy.org. 
En definitiva, aunque en términos generales la política de subvenciones al desguace ha permitido el desguace del $22 \%$ del total de buques, que en términos de capacidad y potencia desguazada con ayudas del IFOP representa el $41 \%$ y $38 \%$, respectivamente, no es menos cierto que este sistema de incentivos para la reducción del esfuerzo pesquero adolece de una gestión adecuada.

\section{SITUACIÓN ACTUAL Y PERSPECTIVAS FUTURAS}

El paso del tiempo sigue mostrando la existencia de una flota pesquera sobredimensionada, en la que existe una desproporción entre los recursos existentes y la capacidad de pesca. Los datos analizados han podido constatar la ineficacia de determinados tipos de ayuda en la reducción del exceso de la capacidad de la flota con el consecuente impacto negativo en los recursos.

En este contexto, no es de extrañar que en la evaluación del impacto de la PPC para el período 2007-2013, la CE haya llegado a la conclusión de que no se han alcanzado los objetivos establecidos y que los instrumentos financieros no han sido eficaces ${ }^{32}$. En particular, pese a la amplia dotación financiera asignada a los planes de desmantelamiento de algunas flotas, no se ha logrado reducir el exceso de capacidad y el índice de descartes sigue siendo elevado. Caso similar es el derivado del análisis de las ayudas destinadas a la vigilancia y control de la actividad pesquera. Surís y Varela (2011), entre otros, consideran que el supuesto fracaso de la PPC en la consecución del equilibrio entre capacidad y recursos no es atribuible totalmente a su política estructural, ya que la misma ha tenido efectos positivos, tanto desde el punto de vista técnico (colaborando a la consecución de los objetivos explícitos de la PPC), como del socioeconómico.

La reforma de la PPC exige que se garantice la adaptación de la flota, asegurando la explotación sostenible de los recursos, respetando el medio ambiente y aplicando medidas socioeconómicas con el fin de minimizar los daños que puedan sufrir los trabajadores que se vean afectados por los planes de ajuste del esfuerzo pesquero.

Cabe esperar, y es deseable, que en un futuro próximo se aplique progresivamente a la gestión pesquera un planteamiento basado en los ecosistemas y se trate de contribuir a la eficacia de las actividades pesqueras en un sector de la pesca y la acuicultura económicamente viable y competitivo, que proporcione un nivel de vida justo y sostenible para aquellas personas que dependen de las actividades pesqueras.

De hecho, la Comisión Europea ha propuesto un nuevo fondo (FEMP) para las políticas marítima y pesquera de la UE para el período 2014-2020 ${ }^{33}$. Dicho

\footnotetext{
${ }^{32}$ Bertrand et al. (2011).

${ }^{33}$ En julio de 2011, la Comisión Europea presentó sus propuestas de reforma de la PPC y entre los objetivos contemplados se encontraban el de "Una Europa que utilizara eficazmente los re-
} 
fondo, que engloba la Política Marítima Integrada ${ }^{34}$ (PMI) pretende facilitar la consecución de los ambiciosos objetivos establecidos en la reforma de la PPC, promover una pesca y acuicultura sostenibles y competitivas y fomentar un desarrollo territorial equilibrado e integrador de las zonas pesqueras (Comisión Europea, 2013) ${ }^{35}$.

La consecución de los objetivos del FEMP contribuirá a la estrategia Europa 2020 (Comisión Europea, 2010) para un crecimiento inteligente, sostenible e integrador y se lograrán a través de las seis prioridades siguientes: Aumentar el empleo y la cohesión territorial mediante el fomento del crecimiento económico, la inclusión social, la creación de empleo y la movilidad laboral en las regiones costeras dependientes de la pesca y acuicultura, así como la diversificación de las actividades pesqueras; impulsar y fomentar una acuicultura y un sector pesquero innovador, competitivo, sostenible y eficiente (especialmente de la pesca costera artesanal); favorecer la aplicación de la PPC mediante la recopilación de datos y la aportación de conocimientos científicos, así como el apoyo al control y la observancia, el fomento de la capacidad institucional y una administración pública eficiente.

En un contexto más local, las pesquerías artesanales y la acuicultura se beneficiarán de la ecologización presupuestaria de la PPC, ya que este nuevo fondo no invertirá más fondos en la construcción de grandes buques. La UE considera a las flotas artesanales como el principal sostén económico en muchas zonas costeras y por ello pretende intensificar las ayudas destinadas a ellas. La propuesta de dotación asciende a 6.500 mill. $€$ para el período comprendido entre 2014 y 2020.

E1 FEMP será un instrumento fundamental para la reforma de la PPC ya que la transición hacia la sostenibilidad exige algunos cambios importantes en la manera en que se pesca y el fondo aportará a los pescadores el estímulo que necesitan, de modo que se ataje la sobrepesca, las poblaciones de peces se recuperen de su declive y la pesca sea menos perjudicial para los ecosistemas marinos. De hecho, el FEMP ayudará a las comunidades altamente dependientes de la pesca a encontrar formas innovadoras de añadir valor a sus capturas y diversi-

cursos" (Comisión Europea, 2011); posteriormente, en diciembre de ese mismo año, propuso el FEMP.

${ }^{34}$ Dada la estrecha interacción entre el sector pesquero y otros sectores marítimos, la PMI aborda las interacciones entre todas las políticas de la UE y los asuntos marítimos, la Comisión presenta en 2007 una propuesta de Política Marítima Integrada para la UE conocida como el Libro Azul (Comisión Europea, 2007).

${ }^{35}$ De hecho, la Comisión Europea (2011) establece que la aplicación de la PPC debe tener en cuenta las interacciones con otros asuntos marítimos, abordados en la PMI, y reconocer la interrelación entre todas las cuestiones relacionadas con los mares y océanos de Europa, incluida la ordenación del espacio marítimo. 
ficar su economía, mientras se da tiempo a las poblaciones de peces a recuperarse aminorando la presión sobre éstas.

En lo que respecta a las ayudas al desguace, a pesar de los 1.700 mill. $€$ invertidos en incentivar el desguace desde la década de 1990, la flota de la UE sigue adoleciendo de exceso de capacidad, al quedar compensado el desguace de buques por la actualización tecnológica de la flota restante que ha dotado a los buques de una mayor capacidad predatoria. Por tanto, el nuevo fondo no destinará más ayudas al desguace e invertirá los fondos comunitarios en actividades productivas desde un punto de vista económico y social, utilizando la diversificación como eje fundamental de estas políticas.

El presupuesto total se asignará a los EEMM según la importancia del sector pesquero en cada país. A partir de ahí, se aplicarán los principios de la gestión compartida entre la Comisión y éstos. De esta manera, los EEMM serán los encargados de elaborar un Programa Operativo para el período donde se especificará cómo se proponen aplicar el dinero que se les ha asignado. Una vez aprobado el programa por parte de la Comisión, cada EEMM decide qué proyectos reciben la financiación. La supervisión de la subvencionabilidad de las medidas de apoyo y la ejecución del programa será por parte de los EEMM y la Comisión.

En definitiva, el nuevo fondo pretende contribuir eficazmente a la consecución de los nuevos objetivos prioritarios, entre los que se encuentran el establecimiento de concesiones de pesca transferibles, la prohibición de los descartes y la consecución del rendimiento máximo sostenible.

\section{CONCLUSIONES}

Aunque la Pesca desempeña un papel relevante para las economías de algunos EEMM de la UE entre los que se encuentra España, el grado de sobreexplotación en muchas poblaciones pesqueras hace que el sector pesquero se enfrente a un futuro incierto como consecuencia de la disminución de las capturas y la pérdida de rentabilidad del sector.

Desde los pioneros trabajos de McIntosh (1899) y Huxley (1881) en los que se defendía la creencia de los recursos marinos como prácticamente inagotables hasta la actualidad se ha recorrido un largo camino. Ya a comienzos del siglo $\mathrm{XX}$ se empezaron a atisbar las consecuencias de un ejercicio irresponsable de la pesca amparado en la creencia de que los recursos pesqueros eran inagotables. Desde los trabajos de H. Scott Gordon (1954) donde se sentaron las bases para la consecución, al menos teórica, de una explotación socialmente óptima, hasta nuestros días, parece que hemos aprendido poco, pues el éxito de muchas de las medidas de gestión puestas en marcha durante los últimos decenios no han conseguido frenar la carrera competitiva por hacerse con la mayor cantidad de re- 
curso con el menor coste posible y colocando a la sobreexplotación pesquera como problema de primera magnitud.

En todo el análisis expuesto en este trabajo subyace la controversia sobre la idoneidad de las subvenciones como instrumento de política económica que pueda garantizar, en primera instancia, la supervivencia de la actividad pesquera y a continuación la explotación racional de los recursos pesqueros basada en la sostenibilidad. Aunque en el caso de la pesca la utilización de diferentes tipos de subsidios tiene una larga historia y a pesar de que tradicionalmente muchos se habían esforzado por destacar sus efectos beneficiosos, en la actualidad se plantean serias dudas sobre la idoneidad en el mantenimiento de estas políticas a largo plazo, poniéndose de manifiesto serios problemas en cuanto a su eficacia y a la posibilidad de control así como su relación con la sobrepesca, como indicaba el informe publicado por la FAO (2003). Por ello, es crucial diferenciar las ayudas que contribuyen a la conservación del medio ambiente de aquellas que pueden conducir a la sobrecapacidad.

El análisis de las subvenciones pesqueras en España y la evolución durante el período 1994-2012 ha puesto de manifiesto que algunos instrumentos financieros no han sido eficaces. Así, se ha destacado una amplia dotación financiera asignada a las modalidades de ayudas clasificadas como malas, en concreto, 2.464 mill. $€$ en el período 1994-2006, según la clasificación del CIP. No obstante esta dotación se reduce considerablemente a menos de la mitad, en concreto 1.125 mill. $€$ si tenemos en cuenta la clasificación de UNEP, WWF y OCDE en cuanto a ayudas negativas.

En este contexto, las ayudas al desguace no han logrado reducir convenientemente el exceso de capacidad pesquera. El estudio revela un uso inadecuado de las ayudas al desguace, ya que se ha podido identificar un número considerable de buques que han recibido ayudas públicas al desguace al poco tiempo de haber recibido ayudas a la modernización.

\section{REFERENCIAS BIBLIOGRÁFICAS}

APEC. (2000): Study into the nature and extent of subsidies in the fisheries sector of APEC member economies. PricewaterhouseCoopers report no. CTI 07/99T. pp. 1228.

ARNASON, R. (2002): Community fisheries management possible applications to Mediterranean fisheries, Conference on Fishery Management and Multi-level Decision Systems. The Mediterranean Case, Salerno, Italy, October 18-19.

ARNASON, R. (2007): Advances in Fisheries Modelling. XVIIIth biannual EAFE conference, Reykjavik, July 9-11. 
BERTRAND, A., CASTELlA, C., FARRANT, T., AVIAT, D., RENAULT, S., AFANASJEVA, A. (2011): Interim evaluation of the European Fisheries Fund (20072013) http://ec.europa.eu/fisheries/documentation/studies/eff_interim_evaluation_en. pdf.

CARDENETE FLORES, M. A., DELGADO LÓPEZ M. C. Y FUENTES SAGUAR, P.D. (2013): "Análisis del impacto de los Fondos Europeos en Andalucía: 2000-2006". Estudios de Economía Aplicada, 31(1): pp. 1-26.

COMISIÓN EUROPEA (1998): La política pesquera común, Luxemburgo.

COMISIÓN EUROPEA (2002): The Reform of the Common Fisheries Policy: Giving EU fisheries sector a future. EC Memo/02/111. May 2002.

COMISIÓN EUROPEA (2007): Una Política Marítima Integrada para la Unión Europea. Bruselas COM, 2007, 575 final.

COMISIÓN EUROPEA (2009a): Informe anual sobre la aplicación del Fondo Europeo de Pesca (2007), Bruselas.

COMISIÓN EUROPEA (2009b): Green Paper Reform of the Common Fisheries Policy. Brussels COM, 2009, 163 final.

COMISIÓN EUROPEA (2010): Europa 2020. Una estrategia para un crecimiento inteligente, sostenible e integrador. Bruselas COM, 2010, 2020 final.

COMISIÓN EUROPEA (2011): Propuesta de Reglamento del Parlamento Europeo y del Consejo sobre la Política Pesquera Común. Bruselas COM, 2011, 425 final.

COMISIÓN EUROPEA (2013): Propuesta modificada de Reglamento del Parlamento Europeo y del Consejo relativo al Fondo Europeo Marítimo y de Pesca. Bruselas COM, 2013, 245 final.

CORDÓN, E. Y GARCÍA, F. (2010): "Evaluación paramétrica y no paramétrica del desempleo pesquero en Marruecos: Una aplicación para el caso andaluz", Revista de Estudios Regionales, 1, pp. 17-44.

COX, A. Y SCHMIDT, C-C. (2002): Subsidies in the OECD fisheries sector: a review of recent analysis and future directions. Paris: Directorate of Agriculture, food and fisheries, OECD.

Evaluación de Ecosistemas del Milenio (2005): Ecosystems and human wellbeing: synthesis. Washington, DC, Island Press.

FAO (1992): La Pesca Marítima y el Derecho del Mar: un decenio de cambio. FAO fisheries circular no. 853. Rome: FAO.

FAO (1995): Código de Conducta para la Pesca Responsable. FAO documento técnico de pesca, No. 350(1). Rome: FAO.

FAO (2003): Introducción a las subvenciones pesqueras. Documento técnico de la FAO 437, FAO, Roma http://www.fao.org/docrep/006/y4647s/y4647s04.htm.

FAO (2004): Consulta técnica sobre la utilización de subsidies en el sector pesquero. Iniciativa técnica mundial sobre las subvenciones a la pesca. Roma 30 de junio-2 de julio de 2004. TC SUB/2004/2.

FAO (2005): Examen de la situación de los recursos pequeros mundiales. Documento Técnico de Pesca, No. 457. Roma: FAO.

FISHSUBSIDY (2013). www.fishsubsidy.org.

FROST, H. AND ANDERSEN, P. (2006): "The Common Fisheries Policy of the European Union and fisheries economics", Marine Policy, 30, pp. 737-746. 
GONZÁLEZ LAXE, F. (2002): Desarrollo y estrategia de la pesca europea. $1^{\text {a }}$ edición, Netbiblo, A Coruña.

GONZÁLEZ LAXE, F. (2010): "Dysfunctions in common fishing regulations". Marine Policy, vol. 34, 293-188.

GONZÁLEZ LAXE, F. (2012): "Modelos de gestión de la política pesquera comunitaria: un proceso histórico", Revista FACES, 38-39, pp. 103-132

GORDON, H. S. (1954): The Economic Theory of a Common-Property Resource: The Fishery, Journal of Political Economy, 62, pp. 124-142.

HANNESSON, R. (1998): "The role of economic tools in redefining fisheries management”. En Pitcher, T. J., Hart, P., Pauly, D. (ed.): Reinventing fisheries management (pp. 251-260). Great Britain: Kluwer Academic publisher.

HATCHER, A. (2000): Subsidies for European fishing fleets: the European Community's structural policy for fisheries 1971-1999, Marine Policy, 24, pp. 129-140.

HUXLEY, T. H. (1881): The Herring, Nature.

KHALILIAN, S., FOESE, R., PROELSS, A. AND REQUATE, T., (2010), "Designed for failure: a critique of the Common Fisheries Policy of the European Union", Marine Policy, 34, pp.1178-1182.

MINISTERIO DE AGRICULTURA, PESCA Y ALIMENTACIÓN (2009): Libro Blanco de la Pesca. Madrid: Centro de publicaciones.

MARKUS, T. (2010): Towards sustainable fisheries subsidies: Entering a new round of reform under the Common Fisheries Policy, Marine Policy, 34, pp. 1117-1124.

MCINTOSH, W. C. (1899): Resources of the Sea, Londres.

MILAZZO, M. (1998): Subsidies in world fisheries: a reexamination. World Bank, Fisheries Series Technical Paper 406, Washington DC: The World Bank. wwwwds.worldbank.org/. (March 2008).

MUNRO, G. R., Y SUMAILA, U. R. (2002): The impact of subsidies upon fisheries management and sustainability: the case of the North Atlantic. Fish and Fisheries, 3, pp. 1-18.

OCDE (2000): Transition to responsible fisheries: Economic and policy implications. Paris: Organización para la Cooperación y Desarrollo Económico.

OMC (Organización Mundial del Comercio) (2001): Declaración ministerial de la OMC, DOHA 2001. WT/MIN (01)/DEC/1.

ORGANIZACIÓN DE LAS NACIONES UNIDAS (2002): Declaración de Johannesburgo sobre el Desarrollo Sostenible, Cumbre Mundial sobre el Desarrollo Sostenible, Johannesburgo, 26 de agosto-4 de septiembre 2002, Nueva York, Naciones Unidas.

PENAS, E., (2002): "The fishery conservation policy of the European Union after 2002: towards long-term sustainability". ICES Journal of Marine Science, 64, pp. 588-595.

PENAS, E., (2008): "Los postulados de la revisión de la Política Pesquera Común". En González Laxe, F. (ed.): Lecciones de Economía Pesquera. A Coruña, Netbiblo: 166-187.

PORTER, G. (2002a): Fisheries Subsidies and Overfishing: Towards a Structured Discussion. UNEP. Génova.

PORTER, G. (2002b): Subsidies and the Environment: An Overview of the State of Knowledge, Paper presented to the OECD Workshop on Environmentally Harmful Subsidies, París, 7-8 November. 
PRELLEZO, R., ACCADIA P., ANDERSEN J.L., ANDERSEN B.S., BUISMAN E., LITTLE A., NIELSEN J.R., POOS J.J., POWELL J. Y RÖCKMANN C. (2012): A review of EU bio-economic models for fisheries: The value of a diversity of models, Marine Policy, 36, pp. 423-431.

SUMAILA, U. R. (2003): "A fish called Subsidy". Science and the Environment, 12 (12).

SUMAILA, U. R. Y PAULY, D. (2006): Catching more bait: a bottom-up reestimation of global fisheries subsidies. University of British Columbia, Fisheries Centre Research Reports 14(6), Vancouver. www.fisheries.ubc.ca/.

SUMAILA, U.R., KHAN A., DYCK A., WATSON R., MUNRO G., TYDEMERS P. AND PAULY D. (2010): A Bottom-Up Re-Estimation of Global Fisheries Subisidies (Working Paper \# 2009-11) University of British Columbia. Canada Fisheries Centre. Research Report.

SURÍS REGUEIRO, J. C. Y GARZA GIL, M.D. (2005): "Ayudas estructurales y expectativas de rentabilidad de las flotas pesqueras en la Unión Europea”, Revista española de Estudios Agrosociales y Pesqueros, 208, pp. 141-169.

SURÍS REGUEIRO, J. C., VARELA LAFUENTE, M. M. Y GARZA-GIL, M. D. (2002): "Profitability of the fishing fleet and structural aid in the European Union", Marine Policy, 26, pp. 107-119.

SURÍS REGUEIRO, J. C., VARELA LAFUENTE, M. M. Y GARZA-GIL, M. D. (2011): "Evolution and perspectives of the Fisheries Structural Policy in the European Union", Ocean \& Coastal Management, 54, pp. 593-600.

SURÍS REGUEIRO, J. C., VARELA LAFUENTE, M. M. Y IGLESIAS-MALVIDO, C. (2003): "Effectiveness of the structural fisheries policy in the European Union", Marine Policy, 27: pp. 535-544.

SURÍS REGUEIRO, J. C. AND VARELA LAFUENTE, M. M. (2011): "Efectos de la política estructural pesquera sobre la flota comunitaria en el período 2000-08", Revista de Estudios Agrosociales y Pesqueros, 230, pp. 87-118.

TRIBUNAL DE CUENTAS EUROPEO (2011): Informe Especial $n^{\circ} 12$.

UNEP (2004): Analyzing the resource impact of fisheries subsidies: matrix approach. United Nations Publication. March 2008.

UNEP (2008): Fisheries Subisidies: A Critical Issue for Trade and Sustainable Development at the WTO. United Nations Publication. May 2008.

VILLASANTE, S. (2010): "Global assessment of the European Union fishing fleet: An update", Marine Policy, 34: pp. 663-670.

VILLASANTE, S. Y SUMAILA, U.R. (2010): "Estimating the effects of technological efficiency on the European fishing fleet", Marine Policy, 34: pp. 720-722.

WWF (WORLD WILDLIFE FUND) (2000): Evidence of poor transparency in fishing subsidy programmes. WWF, Washington, D.C. www.worldwildlife.org. (September 2007).

WWF (2002): Turning the Tide on Fishing Subsidies: Can the World Trade Organization Play a Positive Role?, WWF, Washington.

WWF (2003): Subsidios pequeros europeos y el medio ambiente marino (caso español). 
